\title{
HIV Serostatus Disclosure Among Men Who Have Sex with Men in China in the Era of $\mathrm{U}=\mathrm{U}$ and PrEP
}

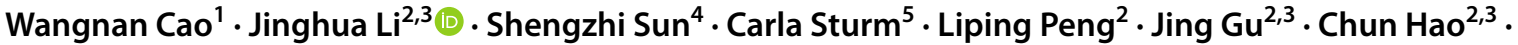 \\ Fengsu $\mathrm{Hou}^{3,6} \cdot$ Dannuo Wei $^{2} \cdot$ Xinyi You ${ }^{2} \cdot$ Yang Deng $^{2} \cdot$ Anna Mia Ekström $^{5}$
}

Accepted: 13 October 2021 / Published online: 25 October 2021

(c) The Author(s) 2021

\begin{abstract}
Given the recent evidence on "Undetectable $=$ Untransmittable" $(\mathrm{U}=\mathrm{U})$ and pre-exposure prophylaxis $(\operatorname{PrEP})$, the present study aimed to investigate HIV disclosure behaviors and their associations with sexual risk behaviors and $\mathrm{U}=\mathrm{U}$ and PrEP awareness among men who have sex with men (MSM) in China. A cross-sectional survey was conducted among 689 MSM recruited through a gay-friendly non-governmental organization located in Chengdu, China in 2018-2019. Information was collected by a structured self-administrated questionnaire. The enrolled sample included 554 (80.4\%) participants who were HIV-negative and 135 (19.6\%) participants with an unknown HIV status. In terms of disclosure, $41.4 \%$ of participants informed all partners about their HIV status all the time (informing behavior), while $30.4 \%$ asked all partners about their HIV status all the time (asking behavior). Only one-fifth knew about $\mathrm{U}=\mathrm{U}$, but this was not statistically associated with either informing or asking behavior. Half (50.5\%) had heard of PrEP but this was not statistically associated with either informing or asking behavior. Common barriers to informing and asking behaviors were lower risk perception of HIV infection, a history of sexually transmitted infections, engagement in receptive sex, and a history of sex with casual partners. We found that both $\mathrm{U}=\mathrm{U}$ and PrEP awareness and HIV serostatus disclosure were infrequent and not associated in this study of Chinese MSM. These data indicate huge information gaps among MSM in China.
\end{abstract}

Keywords HIV $\cdot$ Men who have sex with men $(\mathrm{MSM}) \cdot$ Serostatus disclosure $\cdot$ Undetectable $=$ Untransmittable $(\mathrm{U}=\mathrm{U})$. Treatment as prevention $(\mathrm{Tas} \mathrm{P}) \cdot$ Pre-exposure prophylaxis $(\mathrm{PrEP}) \cdot$ China $\cdot$ Antiretroviral therapy

Jinghua Li

lijinghua3@mail.sysu.edu.cn

1 Department of Social Medicine and Health Education, School of Public Health, Peking University, Beijing 100191, China

2 School of Public Health, Sun Yat-Sen University, North Campus, 74\# Zhongshan 2nd Road, Guangzhou 510000, China

3 Sun Yat-Sen Global Health Institute, Sun Yat-Sen University, Guangzhou, China

4 Department of Environmental Health, Boston University School of Public Health, Boston, MA, USA

5 Department of Global Public Health, Karolinska Institutet, Stockholm, Sweden

6 Department of Public Mental Health, Kangning Hospital, Shenzhen, Guangdong, China

\section{Introduction}

Disclosure of HIV serostatus may bring both advantages and disadvantages. Potential advantages include improved adherence to antiretroviral therapy (ART), psychological relief, and shared decision-making with partners in terms of sex and condom use. Potential disadvantages include stigma and discrimination, voilence, and ending of relationships. Whether or not to disclose one's HIV serostatus is a decision to make after balancing these advantages and disadvantages.

Disclosure of HIV serostatus has been an issue particularly for men who have sex with men (MSM) [1-3], who experience a disproportionate burden of HIV infection [4]. The HIV prevalence among MSM ranges from 5 to $32 \%$ globally [5, 6], and has increased among Chinese MSM from $1 \%$ in $2005,7 \%$ in 2012, and $10 \%$ in 2017 [4, 7-9]. Studies have identifed multiple level of factors associated with HIV disclosure among MSM, including individual factors (e.g., age, illness severity, awareness of HIV status, treatment 
status), interpersonal factors (e.g., type of the partner, intimacy), and social factors (e.g., social support, stigma against people living with HIV as well as homophobia) [10-12]. The prevalence of HIV disclosure varies from 12 to $53 \%$ among MSM living with HIV [13].

Awareness of Undetectable $=$ Untransmittable $(\mathrm{U}=\mathrm{U})$ might reduce the need and motivation to disclose HIV status to partners because it is now established that there is no risk of HIV transmission when the viral load is undetectable [14-18]. The 2019-2020 Positive Perspectives Study reported a very high overall awareness of $88 \%$ for $\mathrm{U}=\mathrm{U}$, regardless of sexual orientation, among people living with HIV in 25 countries including China (50 out of the total 2389 participants), where stigma and discrimination related to lesbian, gay, bisexual and transgender (LGBT) and against people living with HIV is common [19, 20]. However, to our knowledge, there are no studies on the awareness of $\mathrm{U}=\mathrm{U}$ among MSM in China, nor on whether $\mathrm{U}=\mathrm{U}$ influences HIV disclosure behavior.

Pre-exposure prophylaxis (PrEP) offers an excellent option for HIV prevention among MSM at high risk of HIV infection [21, 22]. China supports PrEP usage among MSM but has not been integrated into a nation-wide program [23]. MSM in China show interest in PrEP but also express concerns of its side effects, associated stigma, and costs. The awareness of and access to PrEP might reduce the need and motivation to discuss HIV status with partners because they may assume it is the partner's responsibility to use PrEP to protect them from HIV infection. To the best of our knowledge, no previous studies have been conducted among MSM in China to explore if PrEP awareness and/or usage could affect HIV disclosure behavior.

This study aimed to investigate the prevalence and emerging factors associated with HIV serostatus disclosure among MSM living in Chengdu, China in the era of $\mathrm{U}=\mathrm{U}$ and PrEP. Tangling these associations would increase awareness on highly effective HIV preventive strategies ( $\mathrm{U}=\mathrm{U}$ and $\mathrm{PrEP}$ ) and inform MSM to make reasonable and beneficial decisions in terms of HIV disclosure. Along with other potential determinants of disclosure (e.g., risk perception, substance use, condom use), we hypothesized that awareness of $\mathrm{U}=\mathrm{U}$ and PrEP would be associated with HIV serostatus disclosure behaviors among MSM in the Chinese setting where people living with HIV and sexual minorities are both stigmatized.

\section{Methods}

\section{Study Design and Setting}

We conducted a cross-sectional survey between November 2018 and April 2019 among MSM living in Chengdu,
China. Chengdu was chosen as the study site because it is a city in western China presenting a higher than average HIV prevalence among MSM for decades [24]. At the time of the study, PrEP was not covered by any health insurance. The market price of PrEP was about US \$290 per month, corresponding to $30 \%$ of an average monthly salary among residents living in urban Chengdu [25].

\section{Participants and Recruitment}

Participants were recruited among users of a local gayfriendly non-governmental organization (NGO) whose customers had previously agreed to be contacted for a research purpose. The research team contacted all potential participants from the center's list of customers by phone to screen for eligibility (assigned male sex at birth, aged 18 years or older, and reported having engaged in anal intercourse with at least one man in the previous six months). A total of 890 individuals were found to be eligible and were invited to visit the NGO to complete a digital self-administered anonymous questionnaire, but 170 individuals declined due to a busy schedule, nconvenience of transportation or concerns on privacy. The remaining 720 individuals agreed to come, of which 711 participants completed the survey in person (response rate of $79.9 \%$ ) during the study period. Two male experienced fieldworkers from the collaborating NGO were hired to facilitate the screening and recruitment process. A trained research assistant (a graduate student major in Public Health) from the research group conducted anonymous data collection and quality control.

Before completing the anonymous self-administered survey, participants were briefed about the study purpose and were asked to provide written informed consent. The questionnaire took an average of 20 minutes to complete with a table computer (an iPad), and completeness and logic errors were automatically checked. Participants were reimbursed US $\$ 8$ in cash to compensate for their time spent on the study. Ethical approval was obtained from the Ethics Committee of Sun Yat-sen University ([2018] 049).

\section{Measures}

All survey questions used in the survey were pilot-tested on 15 college students, three MSM peer leaders from the center, and 25 eligible MSM volunteers (who were not invited for the subsequent formal survey). Minor revisions were made based on the pilot results and comments from participants.

\section{Background}

Socio-demographic information collected included age, ethnicity, education, marital status, personal income, 
employment status, and local residence. Participants were also asked about sexual orientation and the age of first sexual intercourse with another man.

\section{HIV Serostatus Disclosure}

HIV serostatus disclosure is an interactive process of asking about a partner's HIV status (asking) and informing the partner about one's own HIV status (informing). We used two separate items (inform and ask) to assess participants' disclosure status: "In the past month, I informed my partners about my own HIV status" and "In the past month, I asked my partners about their HIV status." Response options included: never, occasionally, half of the time, most of the time, and always. Two separate outcome variables were constructed, including "Always informing partners my HIV status" and "Always asking partners about their HIV status." Participants who responded with "always" were classified as $\mathrm{Y}=1$, while the rest were classified together as $\mathrm{Y}=0$. We used these two outcomes as dependent variables and analyzed for independent determinants.

\section{Awareness of $\mathrm{U}=\mathrm{U}$ and PrEP}

We asked participants to determine if the statement " $A$ person with undetectable viral load cannot transmit HIV to others" was correct. Response options included: correct, wrong, and I don't know. Those who responded "wrong" or "I don't know" were combined as having no $\mathrm{U}=\mathrm{U}$ awareness.

\section{PrEP Awareness and Usage}

Participants were asked whether they had heard of any types of PrEP (daily oral PrEP, on-demand oral PrEP, and longacting injectable PrEP) before the survey. Participants who responded affirmatively to any of these three types of PrEP were classified as having PrEP awareness; those who had never heard of any types of PrEP were classified as having no PrEP awareness. For those who had heard of PrEP, we further asked whether they had used (or were using) it; then they were classified as PrEP users or PrEP non-users accordingly.

\section{Other Variables}

HIV status and HIV testing. Participants were asked about their HIV testing history and HIV status (positive, negative, unknown) at the time of the survey. Each participant was offered to take a free HIV test at the center after completing the survey to confirm their HIV status. However, the present analyses used their self-reported data on HIV status because we believe this is more relevant to disclosure. We also asked about their history of sexually transmitted infections (STIs).
Risk perception of HIV infection. Participants who were HIV-negative or had unknown serostatus were asked to rate their perceived risk of HIV infection over the next 6 months using a five-point Likert scale from 1 (very high) to 5 (very low). Responses of very high and high were combined into the higher risk perception group, while responses of very low, low, and neutral were combined into the lower risk perception group.

Positive attitudes towards living with HIV. We constructed three items to estimate if the participants had positive attitudes towards living with HIV, including "people living with HIV can be healthy", "immediate treatment is most effective", and "people living with HIV can have a normal life expectancy." Responses included agree (score 1) versus disagree (score 0 ). A summary score was calculated, with a higher score indicating more positive attitudes towards living with HIV.

Sexual behaviors. Participants were asked to recall the total number of partners with whom they had had sex in the past month. For each partner, we asked whether the partnership was regular or casual, whether a condom was used (yes, no), whether drugs were used during sex (yes, no), the venue used to find this partner (online, physical setting, both), and the participant's role in the sexual intercourse with this partner (insertive only, receptive only, both insertive and receptive). Participants who reported having had sex with more than one partners in the past month were classified as having "multiple sexual partnerships." Participants who did not use condoms with all partners in the past month were classified into "inconsistent condom use".

\section{Statistical Analysis}

Univariate associations were assessed using binary logistic regression to examine each of the independent variables listed above with the two outcomes of "Always inform partners my HIV status" and "Always asking partners about their HIV status". Subsequently, significant variables $(\mathrm{p}<0.05)$ from the univariate analyses and three variables ( $\mathrm{U}=\mathrm{U}$ awareness, PrEP awareness, and self-reported HIV status; regardless of the univariate analyses results) were included in multivariate logistic regression analyses. The measures of association are presented as unadjusted odds ratio $(\mathrm{ORu})$ versus adjusted odds ratio (AOR), with 95\% confidence intervals. All statistical analyses were performed using IBM SPSS Statistics (version 25), and two-tailed $\mathrm{p}<0.05$ was considered statistically significant. 


\section{Results}

\section{Descriptive Characteristics}

\section{Background Characteristics}

Of the 711 participants who completed the survey, 554 reported being HIV-negative, 135 reported not knowing their status, and 22 reported being HIV-positive. Due to the small sample size of MSM known to be living with HIV in the present study, we are unable to perform a subgroup analysis by HIV status in terms of predictors of disclosure. Those 22 HIV-positive participants were excluded from the final analyses because people with different HIV status would have different motivations, repercussions, and communication challenges in terms of HIV disclosure.

The characteristics of the 689 participants who were HIV-negative or unknown are presented in Table 1 (Table 1). The mean age of the participants was 30 years $(\mathrm{SD}=11)$, and $57.3 \%$ had attended college or above. Half $(54.0 \%)$ of the participants were single, $13.4 \%$ were married to woman, and $26.0 \%$ were in a stable relationship with a boyfriend. The majority of the participants selfreported their sexual orientation as homosexual (75.9\%), and $19.0 \%$ self-reported as bisexual, which was more prevalent among married participants. More than half of the participants (54.9\%) reported having had their first homosexual intercourse before age 21 years (Table 1).

\section{HIV Serostatus Disclosure Status}

Just over forty percent (41.8\%) of participants always informed their partners about their HIV status, while $14.5 \%$ never did. Almost one-third (30.8\%) always asked all partners about their HIV status, while $13.6 \%$ never asked (Table 1). Combining the two outcomes, 179 participants (25.4\%) always asked and informed about HIV status, 57 (8.3\%) never informed nor asked about HIV status, and 453 (65.7\%) participants were in between. These two disclosure behaviors were correlated with each other (Pearson correlation coefficient: 0.786, $\mathrm{p}<0.01$ ).

\section{$\mathrm{U}=\mathrm{U}$ and PrEP Awareness/Usage}

Only $20 \%$ of participants knew about $\mathrm{U}=\mathrm{U}$. Approximately half of the participants $(50.8 \%)$ had heard about PrEP before the study, and the awareness was $32.7 \%$, $29.8 \%$, and $6.2 \%$ for daily oral PrEP, on-demand PrEP, and long term injecting PrEP, respectively. None of the participants had used (or were using) any types of PrEP.
Table 1 Background characteristics and HIV serostatus disclosure status of the participants $(\mathrm{N}=689)$

\begin{tabular}{|c|c|c|}
\hline Items & $\mathrm{N}$ & $\%$ \\
\hline \multicolumn{3}{|c|}{ Background characteristics } \\
\hline \multicolumn{3}{|l|}{ Age (years) } \\
\hline$\leq 25$ & 311 & 45.1 \\
\hline$>25$ & 378 & 54.9 \\
\hline \multicolumn{3}{|l|}{ Ethnicity } \\
\hline Han & 668 & 97.0 \\
\hline Others & 21 & 3.0 \\
\hline \multicolumn{3}{|l|}{ Local residence } \\
\hline No & 43 & 6.2 \\
\hline Yes & 646 & 93.8 \\
\hline \multicolumn{3}{|c|}{ Highest education obtained } \\
\hline Below than university & 294 & 42.7 \\
\hline University or above & 395 & 57.3 \\
\hline \multicolumn{3}{|l|}{ Relationship status } \\
\hline Single & 3872 & 54.0 \\
\hline Married to a woman & 92 & 13.4 \\
\hline Having boyfriends & 179 & 26.0 \\
\hline Divorced/widow/others & 46 & 6.7 \\
\hline \multicolumn{3}{|l|}{ Employment status } \\
\hline Full time & 438 & 63.6 \\
\hline Part time & 30 & 4.4 \\
\hline Unemployed & 221 & 32.1 \\
\hline \multicolumn{3}{|c|}{ Personal monthly income (USD) } \\
\hline$<423$ & 221 & 32.1 \\
\hline $423-845$ & 254 & 36.9 \\
\hline$>845$ & 214 & 31.1 \\
\hline \multicolumn{3}{|c|}{ Self-identified sexual orientation } \\
\hline Homosexual & 523 & 75.9 \\
\hline Heterosexual & 3 & 0.4 \\
\hline Bisexual & 131 & 19.0 \\
\hline Other & 32 & 4.6 \\
\hline \multicolumn{3}{|c|}{ Age of first homosexual intercourse (years) } \\
\hline$<21$ & 378 & 54.9 \\
\hline$\geq 21$ & 311 & 45.1 \\
\hline \multicolumn{3}{|c|}{ HIV serostatus disclosure status } \\
\hline \multicolumn{3}{|c|}{ I asked all partners in the past month about their HIV status } \\
\hline Never & 94 & 13.6 \\
\hline Occasionally & 150 & 21.8 \\
\hline Half of the time & 66 & 9.6 \\
\hline Most of the time & 167 & 24.2 \\
\hline Always & 212 & 30.8 \\
\hline \multicolumn{3}{|c|}{ I informed all partners in the past month of my HIV status } \\
\hline Never & 100 & 14.5 \\
\hline Occasionally & 115 & 16.7 \\
\hline Half of the time & 43 & 6.2 \\
\hline Most of the time & 143 & 20.8 \\
\hline Always & 288 & 41.8 \\
\hline
\end{tabular}




\section{Other Disclosure-Related Variables}

Around one-fifth (18.3\%) of the participants had never tested for HIV, while the rest $(81.7 \%)$ had tested for HIV at least once. Half of the participants (50.6\%) had tested in the past six months (recent HIV testing). The study found that $15.1 \%$ of participants perceived a high or very high risk of HIV infection, while $58.5 \%$ perceived a low or very low risk. Surprisingly few participants $(7.8 \%)$ reported any history of STI. The mean score of positive attitudes towards living with HIV was $2.45(\mathrm{SD}=0.70)$ out of maximum 3 .

In the past month, $21.0 \%$ of the participants reported having had no partner, $48.2 \%$ reported having sex with one person, and $30.7 \%$ reported having sex with more than one partner (defined as being in multiple sexual partnerships). More than half of the participants (73.7\%) primarily searched for partners through mobile apps (e.g., blued, jack'd), while $20.2 \%$ used physical venues (e.g. bars, baths, parks, parties) only, and $6.1 \%$ used both ways. One-third of the participants (36.6\%) reported inconsistent condom use with all partners in the past month. Around one-quarter of the participants (22.8\%) reported drug use during sex. About half (48.9\%) reported insertive sex only, $34.6 \%$ reported receptive sex only, and $16.5 \%$ reported both. Regarding type of partner, $68.8 \%$ reported sex with regular partners in the past month, and $47.8 \%$ reported sex with casual partners; thus $25.1 \%$ (94/374) of those with regular partners also reported sex with causal ones (Table 2).

\section{Univariate Logistic Regression Analyses Predicting HIV Serostatus Disclosure}

\section{Sociodemographic and HIV Serostatus Disclosure}

Participants were more likely to always inform all partners about their HIV status if they had completed a higher level of education $(\mathrm{ORu}=1.72 ; 95 \% \mathrm{CI} 1.26-2.35)$ or were in a relationship with a boyfriend $(\mathrm{ORu}=2.10 ; 95 \% \mathrm{CI} 1.46-3.01$, compared to single men). Age, as a continuous variable, was negatively associated with always informing all partners about their HIV status $(\mathrm{p}<0.001)$. Older participants ( $>25$ years old) were less likely to always inform all their partners about their HIV status than younger participants $(\mathrm{ORu}=0.45$; 95\% CI 0.33-0.61). Similarly, age of first malemale intercourse (as a continuous variable) was negatively associated with always informing all partners about their HIV status $(p=0.031)$. Participants who reported having their first male intercourse at an older age ( $\geq 21$ years old) were less likely to always inform all partners about their HIV status than those who had their first intercourse with another man at a younger age $(\mathrm{ORu}=0.66$; 95\% CI 0.49-0.90). Participants monthly income was marginally associated with
Table 2 HIV and sexual behavioural characteristics of the participants $(\mathrm{N}=689)$

\begin{tabular}{|c|c|c|}
\hline Items & $\mathrm{N}$ & $\%$ \\
\hline \multicolumn{3}{|l|}{$U=U$ awareness } \\
\hline Yes & 138 & 20.0 \\
\hline No & 551 & 80.0 \\
\hline \multicolumn{3}{|l|}{ PrEP awareness } \\
\hline Yes & 350 & 50.8 \\
\hline No & 339 & 49.2 \\
\hline \multicolumn{3}{|l|}{ Self-reported HIV status } \\
\hline Negative & 554 & 80.4 \\
\hline Unknown & 135 & 19.6 \\
\hline Lifetime HIV testing & 563 & 81.7 \\
\hline Recent HIV testing ( $<$ six months) & 360 & 50.6 \\
\hline A history of STIs (Yes) & 54 & 7.8 \\
\hline \multicolumn{3}{|l|}{ Risk perception of HIV infection } \\
\hline Very low & 170 & 24.7 \\
\hline Low & 233 & 33.8 \\
\hline Neutral & 182 & 26.4 \\
\hline High & 77 & 11.2 \\
\hline Very high & 27 & 3.9 \\
\hline \multicolumn{3}{|l|}{ Number of sexual partners } \\
\hline 0 & 145 & 21.0 \\
\hline 1 & 332 & 48.2 \\
\hline $2-3$ & 178 & 25.8 \\
\hline $4-5$ & 34 & 4.9 \\
\hline Inconsistent condom use (Yes) & 199 & 36.6 \\
\hline Drug use during sex (Yes) & 124 & 22.8 \\
\hline \multicolumn{3}{|l|}{ Sexual roles during anal intercourse } \\
\hline Insertive sex only & 266 & 48.9 \\
\hline Receptive sex only & 188 & 34.6 \\
\hline Both & 90 & 16.5 \\
\hline Had sex with regular partners (Yes) & 374 & 68.8 \\
\hline Had sex with casual partners (Yes) & 260 & 47.8 \\
\hline \multicolumn{3}{|l|}{ Venue for finding new partners } \\
\hline Apps (e.g., blued, jack'd) & 401 & 73.7 \\
\hline Physical setting (e.g., bar, bath, park, party) & 110 & 20.2 \\
\hline Both & 33 & 6.1 \\
\hline
\end{tabular}

Sexual behavior variables were limited to participants who had at least one partner in the past month $(\mathrm{n}=544)$

STI: Sexual Transmitted Infection, $U=U$ : Undetectable $=$ Untransmittable, $\operatorname{PrEP}$ : Pre-exposure Prophylaxis

their informing behavior, while sexual orientation (homosexual vs. bisexual) was not.

Compared with participants who were single, participants who were in a relationship with a boyfriend were more likely to always ask all of their partners about their HIV status $(\mathrm{ORu}=1.73 ; 95 \% \mathrm{CI} 1.19-2.52)$. Age, as a continuous variable, was not significantly associated with always asking all partners about their HIV status. However, older participants ( $>25$ years old) were less likely than their younger 
counterparts to always ask all partners about their HIV status $(\mathrm{ORu}=0.69 ; 95 \%$ CI 0.50-0.96). Compared with participants who earned < US $\$ 423$ (equals to CNY3000) per month, those who earned US \$423-845 (equals to CNY3000-6000) per month had no statistical difference in always asking all partners about their HIV status, but participants who earned > USD845 (equals to CNY6000) were less likely to always ask all partners about their HIV status $(\mathrm{ORu}=0.66$; 95\% CI 0.44-0.98). Participants' level of education was marginally associated with their asking behavior while employment status, sexual orientation, and age at first intercourse with another man were not significantly associated with asking behavior (Table 3).

\section{Association Between $\mathrm{U}=\mathrm{U}$ Awareness and HIV Serostatus Disclosure}

In univariate analyses, being aware of $\mathrm{U}=\mathrm{U}$ was not statistically significantly associated with either always informing partners about one's HIV status $(\mathrm{ORu}=0.90 ; 95 \% \mathrm{CI}$ $0.62-1.32$ ) or always asking partners about their HIV status $(\mathrm{ORu}=0.86$; 95\% CI 0.57-1.30) (Table 3).

\section{Association Between PrEP Awareness and HIV Serostatus Ddisclosure}

PrEP awareness was associated with always informing partners about their HIV status in univariate analysis $(\mathrm{ORu}=1.39$; 95\% CI 1.02-1.88). PrEP awareness was not statistically significantly associated with asking partners about their HIV status in univariate analyses $(\mathrm{ORu}=1.09$; 95\% CI 0.79-1.51) (Table 3).

\section{Associations Between Other Potential Variables and HIV Serostatus Disclosure}

Participants' self-reported HIV status and recent HIV testing were not significantly associated with always informing partners about their HIV status. Positive attitudes towards living with HIV was marginally associated with always informing partners about one's HIV status in univariate analysis $(\mathrm{ORu}=1.23$; 95\% CI $0.99-1.54, \mathrm{p}<0.1)$. A history of STIs was marginally associated with always informing partners about one's HIV status in univariate analysis $(\mathrm{ORu}=0.56$; 95\% CI 0.31-1.03, p <0.1) (Table 3).

Participants who had sex with regular partners were more likely to always inform partners about their HIV status $(\mathrm{ORu}=1.68 ; 95 \% \mathrm{CI} 1.15-2.45)$. Participants were less likely to always inform their partner about their HIV status if they perceived themselves to be at lower risk of HIV infection $(\mathrm{ORu}=0.65 ; 95 \% \mathrm{CI} 0.47-0.88)$, if they reported receptive sex only $(\mathrm{ORu}=0.66$; 95\% CI $0.45-0.96$ vs. those who reported insertive sex only), or reported sex with casual partners in the past 6 months $(\mathrm{ORu}=0.50$; 95\% CI 0.35-0.70). Other variables, such as having multiple sexual partnerships, consistent condom use, drug use during sex, and venue to look for partners were not significantly associated with participants' informing behavior in univariate analyses (Table 3 ).

Participants' self-reported HIV status and positive attitudes towards living with HIV were not significantly associated with always asking partners about their HIV status. Recent HIV testing was significantly associated with always asking partners about their HIV status in univariate analysis $(\mathrm{ORu}=1.56$; 95\% CI 1.13-2.16). A history of STIs was significantly associated with always asking partners about their HIV status in univariate analysis $(\mathrm{ORu}=0.45 ; 95 \%$ CI $0.22-0.91)$ (Table 3$)$.

Participants were more likely to always ask partners about their HIV status if they had sex with regular partners $(\mathrm{ORu}=1.92 ; 95 \%$ CI 1.26-2.91). Participants were less likely to always ask about their partners' HIV status if they perceived themselves to be at lower risk of HIV infection $(\mathrm{ORu}=0.61 ; 95 \% \mathrm{CI} 0.49-0.86)$ and reported having had sex with casual partners $(\mathrm{ORu}=0.44 ; 95 \%$ CI $0.30-0.63)$. Other variables, such as multiple sexual partnerships, consistent condom use, drug use during sex, sexual role during intercourse, and venue to look for partners were not significantly associated with participants' asking behavior in univariate analyses (Table 3).

\section{Multivariate Logistic Regression Analysis Predicting HIV Serostatus Disclosure}

The variables associated with HIV serostatus disclosure in the multivariate analysis are presented in Table 3 and further illustrated in Fig. 1. Six variables were significantly associated with always informing partners about one's HIV status, including age (AOR $=0.48 ; 95 \%$ CI $0.30-0.78,>25$ vs. $\leq 25$ years old), education $(\mathrm{AOR}=1.68 ; 95 \% \mathrm{CI}$ $1.11-2.53$, university or above vs. below than university), risk perception of HIV infection $(\mathrm{AOR}=0.65 ; 95 \% \mathrm{CI}$ $0.47-0.88)$, lower vs. higher risk perception), sexual role during intercourse ( $\mathrm{AOR}=0.48 ; 95 \%$ CI $0.31-0.74$, receptive sex only vs. insertive sex only), having sex with regular partners (AOR $=1.32$; 95\% CI 1.04-2.11), and having sex with casual partners $(\mathrm{AOR}=0.50 ; 95 \%$ CI $0.29-0.87)$ (Table 3).

Three variables that were significantly associated with asking partners about HIV status all the time: recent HIV testing ( $\mathrm{AOR}=1.46 ; 95 \% \mathrm{CI} 1.01-2.14$ ), risk perception of HIV infection $(\mathrm{AOR}=0.61 ; 95 \%$ CI $0.41-0.91$, lower vs. higher risk perception), and having had sex with casual partners $(\mathrm{AOR}=0.55 ; 95 \%$ CI $0.31-0.97)$ (Table 3$)$. 
Table 3 Univariate and multivariate regression analyses of the components associated with HIV serostatus disclosure

\begin{tabular}{|c|c|c|c|c|}
\hline \multirow[t]{2}{*}{ Items } & \multicolumn{2}{|c|}{ Inform partners about one's own HIV status } & \multicolumn{2}{|c|}{ Ask partners about their HIV status } \\
\hline & $\mathrm{ORu}(95 \% \mathrm{CI})$ & AOR $(95 \% \mathrm{CI})$ & $\mathrm{ORu}(95 \% \mathrm{CI})$ & AOR $(95 \% \mathrm{CI})$ \\
\hline \multicolumn{5}{|l|}{ Sociodemographic } \\
\hline \multicolumn{5}{|l|}{ Age (years) } \\
\hline$\leq 25$ & 1.00 & 1.00 & 1.00 & 1.00 \\
\hline$>25$ & $0.45(0.33,0.61)^{* * *}$ & $0.48(0.30,0.78)^{* *}$ & $0.69(0.50,0.96)^{*}$ & $0.84(0.52,1.36)$ \\
\hline \multicolumn{5}{|l|}{ Highest education obtained } \\
\hline Below than university & 1.00 & 1.00 & 1.00 & 1.00 \\
\hline University or above & $1.72(1.26,2.35)^{* * *}$ & $1.68(1.11,2.53) *$ & $1.34(0.60,1.87) \dagger$ & $1.27(0.83,1.94)$ \\
\hline \multicolumn{5}{|l|}{ Relationship status } \\
\hline Single & 1.00 & 1.00 & 1.00 & 1.00 \\
\hline Married to a woman & $0.75(0.46,1.21)$ & $0.89(0.61,2.35)$ & $0.91(0.54,1.53)$ & $0.99(0.50,1.98)$ \\
\hline Having boyfriend & $2.10(1.46,3.01)^{* * *}$ & $1.37(0.86,2.19)$ & $1.73(1.19,2.52)^{* *}$ & $1.23(0.77,1.97)$ \\
\hline \multicolumn{5}{|l|}{ Employment status } \\
\hline Full time & 1.00 & 1.00 & 1.00 & 1.00 \\
\hline Part time & $0.80(0.36,1.74)$ & $0.80(0.30,2.15)$ & $1.52(0.70,3.28)$ & $1.75(0.69,4.46)$ \\
\hline Unemployed & $1.55(1.12,2.15)^{* *}$ & $1.19(0.68,2.08)$ & $1.49(1.05,2.10)^{*}$ & $1.07(0.61,1.85)$ \\
\hline \multicolumn{5}{|l|}{ Personal monthly income (USD) } \\
\hline$<423$ & 1.00 & 1.00 & 1.00 & 1.00 \\
\hline $423-845$ & $0.71(0.49,1.02) \dagger$ & $1.10(0.62,1.95)$ & $0.81(0.56,1.19)$ & $0.95(0.53,1.69)$ \\
\hline$>845$ & $0.71(0.49,1.05) \dagger$ & $0.94(0.50,1.77)$ & $0.66(0.44,0.98)^{*}$ & $0.71(0.37,1.35)$ \\
\hline \multicolumn{5}{|l|}{ Self-identified sexual orientation } \\
\hline Homosexual & 1.00 & - & 1.00 & - \\
\hline Bisexual & $0.94(0.64,1.39)$ & & $1.27(0.85,1.91)$ & \\
\hline \multicolumn{5}{|l|}{ Age of first homosexual intercourse (years) } \\
\hline$<21$ & 1.00 & 1.00 & 1.00 & - \\
\hline$\geq 21$ & $0.66(0.49,0.90)^{* *}$ & $0.68(0.45,1.04)$ & $0.90(0.65,1.25)$ & \\
\hline \multicolumn{5}{|l|}{$U=U$ and $\operatorname{PrEP}$ awareness } \\
\hline \multicolumn{5}{|l|}{$U=U$ awareness } \\
\hline No & 1.00 & 1.00 & 1.00 & 1.00 \\
\hline Yes & $0.90(0.62,1.32)$ & $1.03(0.64,1.65)$ & $0.86(0.57,1.30)$ & $1.05(0.65,1.70)$ \\
\hline \multicolumn{5}{|l|}{ PrEP awareness } \\
\hline No & 1.00 & 1.00 & 1.00 & 1.00 \\
\hline Yes & $1.39(1.02,1.88)^{*}$ & $1.34(0.91,1.98)$ & $1.09(0.79,1.51)$ & $0.96(0.64,1.42)$ \\
\hline \multicolumn{5}{|l|}{ HIV-related variables } \\
\hline \multicolumn{5}{|l|}{ Self-reported HIV status } \\
\hline Unknown & 1.00 & 1.00 & 1.00 & 1.00 \\
\hline Negative & $0.91(0.62,1.33)$ & $0.76(0.45,1.29)$ & $1.34(0.88,2.05)$ & $1.55(0.90,2.67)$ \\
\hline \multicolumn{5}{|l|}{ Recent HIV testing (<6 months) } \\
\hline No & 1.00 & - & 1.00 & 1.00 \\
\hline Yes & $1.08(0.80,1.45)$ & & $1.56(1.13,2.16)^{* *}$ & $1.46(1.01,2.14)^{*}$ \\
\hline Positive attitudes towards living with HIV & $1.23(0.99,1.54) \dagger$ & $1.14(0.86,1.52)$ & $0.98(0.78,1.23)$ & - \\
\hline \multicolumn{5}{|l|}{ A history of STI } \\
\hline No & 1.00 & 1.00 & 1.00 & 1.00 \\
\hline Yes & $0.56(0.31,1.03) \dagger$ & $0.65(0.31,1.36)$ & $0.45(0.22,0.91)^{*}$ & $0.63(0.29,1.39)$ \\
\hline \multicolumn{5}{|l|}{ Risk perception of HIV infection } \\
\hline High/very high & 1.00 & 1.00 & 1.00 & 1.00 \\
\hline Very low/low/neutral & $0.65(0.47,0.88)^{* *}$ & $0.60(0.40,0.89)^{*}$ & $0.61(0.49,0.86)^{* *}$ & $0.61(0.41,0.91)^{*}$ \\
\hline \multicolumn{5}{|l|}{ Sexual behaviors } \\
\hline Multiple sex partnership & & & & \\
\hline
\end{tabular}


Table 3 (continued)

\begin{tabular}{|c|c|c|c|c|}
\hline \multirow[t]{2}{*}{ Items } & \multicolumn{2}{|c|}{ Inform partners about one's own HIV status } & \multicolumn{2}{|c|}{ Ask partners about their HIV status } \\
\hline & $\mathrm{ORu}(95 \% \mathrm{CI})$ & AOR $(95 \% \mathrm{CI})$ & $\mathrm{ORu}(95 \% \mathrm{CI})$ & AOR $(95 \% \mathrm{CI})$ \\
\hline No & 1.00 & - & 1.00 & - \\
\hline Yes & $0.72(0.51,1.02)$ & & $0.73(0.50,1.07)$ & \\
\hline \multicolumn{5}{|c|}{ Consistent condom use } \\
\hline No & 1.00 & - & 1.00 & - \\
\hline Yes & $0.88(0.62,1.24)$ & & $1.06(0.73,1.54)$ & \\
\hline \multicolumn{5}{|l|}{ Drug use during sex } \\
\hline No & 1.00 & - & 1.00 & - \\
\hline Yes & $0.96(0.64,1.45)$ & & $1.27(0.83,1.94)$ & \\
\hline \multicolumn{5}{|c|}{ Sexual role during intercourse } \\
\hline Insertive sex only & 1.00 & 1.00 & 1.00 & - \\
\hline Receptive sex only & $0.66(0.45,0.96)^{*}$ & $0.48(0.31,0.74) * * *$ & $0.74(0.49,1.01)$ & \\
\hline Both & $0.56(0.34,0.92)^{*}$ & $0.49(0.28,0.84)^{* *}$ & $0.89(0.52,1.45)$ & \\
\hline \multicolumn{5}{|c|}{ Had sex with regular partners } \\
\hline No & 1.00 & 1.00 & 1.00 & 1.00 \\
\hline Yes & $1.68(1.15,2.45)^{* *}$ & $1.32(1.04,2.11)^{*}$ & $1.92(1.26,2.91)^{* * *}$ & $1.06(0.57,1.97)$ \\
\hline \multicolumn{5}{|c|}{ Had sex with casual partners } \\
\hline No & 1.00 & 1.00 & 1.00 & 1.00 \\
\hline Yes & $0.50(0.35,0.70)^{* * *}$ & $0.50(0.29,0.87)^{*}$ & $0.44(0.30,0.63)^{* * *}$ & $0.55(0.31,0.97)^{* *}$ \\
\hline \multicolumn{5}{|c|}{ Venue to know partners } \\
\hline Apps & 1.00 & - & 1.00 & 1.00 \\
\hline Physical setting & $0.99(0.65,1.52)$ & & $0.92(0.58,1.44)$ & $0.86(0.53,1.40)$ \\
\hline Both & $0.87(0.42,1.79)$ & & $0.36(0.14,0.96)^{*}$ & $0.45(0.16,1.25)$ \\
\hline
\end{tabular}

STIs: sexually transmitted infections, ORu: univariate odds ratio, STI: Sexual Transmitted Infection, $U=U$ : Undetectable $=$ Untransmittable, PrEP: Pre-exposure Prophylaxis, AOR: adjusted odds ratio

${ }^{a}$ Sexual behaviors among participants who had at least one partner in the past month $(\mathrm{n}=544)$

${ }^{\dagger} \mathrm{P}<0.10, * \mathrm{P}<0.05, * * \mathrm{P}<0.01, * * * \mathrm{P}<0.001$

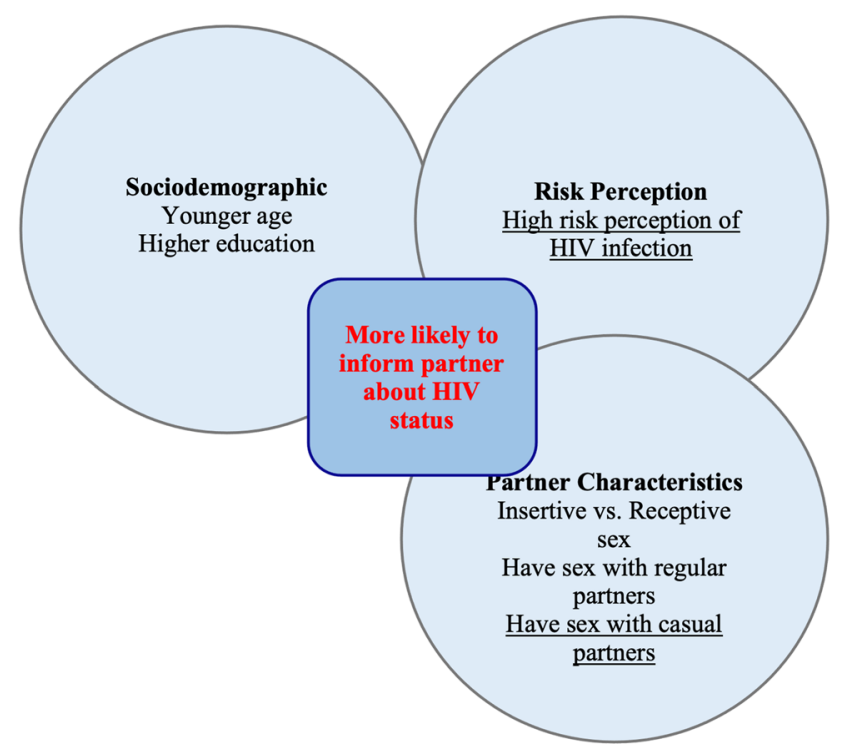

Fig. 1 Significant variables for HIV serostatus disclosure among men who have sex with men in Chengdu, China. Variables presented in the figure were statistically significant at the 5\% level in multivari-

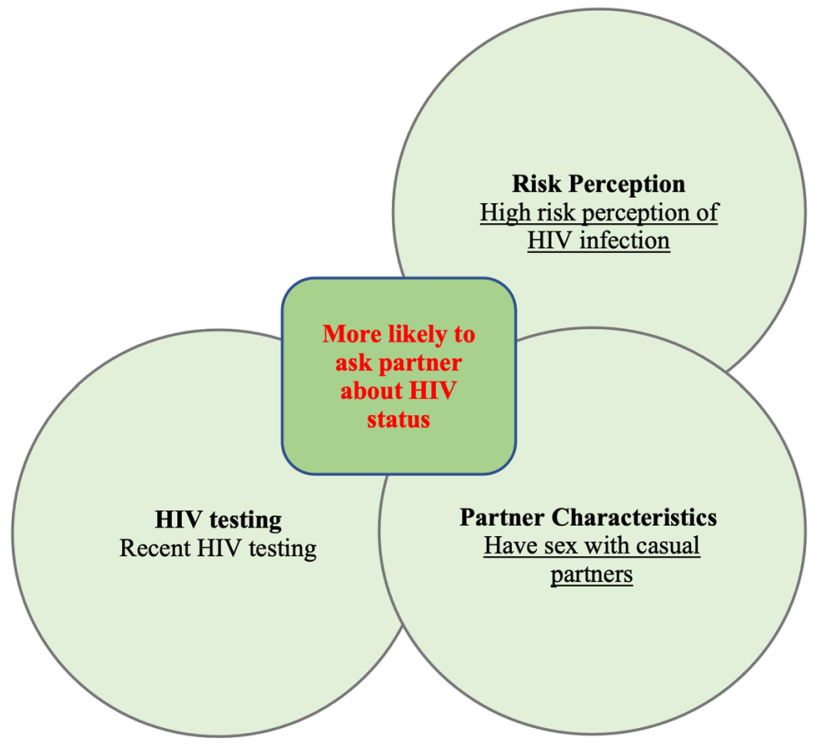

ate models (Table 3), those underlined variables were significant variables for both informing and asking behaviors. 


\section{Discussion}

We found low rates of always asking and informing about HIV status among Chinese MSM. This was not due, however, to high awareness of $\mathrm{U}=\mathrm{U}$ and PrEP, but rather the opposite because the awareness of treatment as prevention was very low. Half of the participants had tested for HIV in the past 6 months, which was associated with asking more about their partner's HIV status. Common barriers to HIV disclosure identified in this sample included perceiving a low risk of HIV infection, engaging in receptive sex, and having sex with casual partners. Compared with MSM who did not know their HIV status, HIV-negative MSM reported a similar level of either asking or informing behaviors.

We did not find any association between the $\mathrm{U}=\mathrm{U}$ awareness and disclosure behavior. The unexpected low level of $\mathrm{U}=\mathrm{U}$ awareness indicates a huge information gap among Chinese MSM in Chengdu, when compared with MSM in western countries (e.g., Australia, USA) [26, 27], which is in line with Asian men being less aware of $\mathrm{U}=\mathrm{U}$ compared to Caucasian MSM in a mixed ethnic population in the U.S. [28]. We did not find relevant data for MSM living in other Asian countries for a comparison here. The low awareness might be explained by a lack of advertisements and information about $\mathrm{U}=\mathrm{U}$ in China, mistrust of the information and its source, and limited availability of viral load testing. In routine HIV care, all patients newly diagnosed with HIV receive ART initiation counseling, in which the counselor mentions $\mathrm{U}=\mathrm{U}$ as one of the advantages of ART for those who are married or in a stable relationship regardless of sexual orientation. Thus, today, MSM living without HIV would have no or very limited opportunities to get the $\mathrm{U}=\mathrm{U}$ information from a health professional, i.e., the source of information that most deem as highly credible. Grassroot non-governmental organizations also play a key role in delivering HIV prevention and treatment programs for MSM in China, but they mostly focus on HIV testing and referral for ART. They generally lack skilled professionals, and thereby also the credibility to deliver the $\mathrm{U}=\mathrm{U}$ information [29]. As a result, awareness of $U=U$ has not spread very widely in the Chinese MSM community. In addition, under the current health and service system in China (Chengdu and elsewhere), viral load testing is provided free of charge only once a year to those on ART for at least 6 months. For most regions, the biggest barrier to viral load testing is the high cost of the assay (US \$75 per test) and the lack of sophisticated laboratories and experienced professionals [30].

We did not find any association between PrEP awareness and disclosure behavior. PrEP is offered as an alternative to other HIV preventive strategies and in various forms (daily or on-demand oral PrEP and longacting injectable PrEP) to meet varying individual needs. Although the hypothetical willingness of PrEP use was high among Chinese MSM if provided for free [23], it is worth noting that PrEP remains inaccessible to most for different reasons, including lack of a supporting national guideline, constrained resources allocated to PrEP, limited trained health professionals in providing PrEP, and high out-of-pocket costs (30\% of an average monthly salary) [31, 32]. An increased PrEP awareness may enable MSM to talk more openly about HIV status. However, the association between actual PrEP use and disclosure requires further investigation. We were unable to test this association because none of our participants had any experience of taking PrEP.

A low risk perception of HIV infection was a common barrier to both asking and informing about HIV status, which was consistent with the current disclosure literature among MSM [33, 34]. For example, perceiving a lower risk of HIV infection was associated with less self-disclosure among 1,044 gay and bisexual men in Australia [34]. One approach to deal with inadequate risk perceptions may be to emphasize routine HIV testing [35]. Given that only half of the participants in the current study had tested for HIV in the past 6 months, more efforts are needed to increase access to HIV testing.

MSM engaging in receptive sex were less likely to ask their partners about their HIV status, possibly explained by a lower hierarchical position and less negotiating power in terms of initiating safer sex [36]. However, since receptive MSM have approximately 2 to 6 times higher risk of HIV infection than insertive MSM [37], tailored interventions, such as communication training and empowerment programs, should be provided to all MSM regardless of sexual preference to enhance safer sex behaviors.

The HIV prevalence of $3 \%$ in our study was lower than the rates reported among Chengdu MSM in previous studies (e.g., 16\% between 2009 and 2014) [38, 39]. The relatively low HIV prevalence might be explained by the recruiting strategy and characteristics of the selected sample. We recruited participants from a local gay-friendly non-governmental organization (Chengdu Tongle Health Counselling Service Center), which mainly provides HIV prevention services and limited HIV treatment services, due to its scope and staff expertise. MSM living with HIV come to the Center to participate in gay community activities and psychological support programs, but most receive their ART at the local hospital and CDC clinic. Thus MSM without known HIV would be more likely to attend the center e.g., for regular HIV testing, making them more likely to be "captured" by our in-person questionnaire. Moreover, the relatively low HIV prevalence could also be explained by the participants being young (half were $<25$ years old) and 
higher educated (56\% had a bachelor's degree) than the average. We found that younger MSM and those with a higher education were more likely than their counterparts to engage in both asking and informing behaviors related to HIV disclosure [40]. Thus, the current disclosure level (30-41\%) might be an over-optimistic estimation of the local Chinese MSM population as a whole.

The present study is subject to several limitations. First, participants were recruited through a local NGO, and only MSM who had connections to the local NGO were available for recruitment into this study. Because this NGO has a clientele of MSM that likely are relatively more open about their sexual identity, more educated, have higher incomes, and are younger than average, our findings might not be generalizable to older and less educated MSM or MSM living in more rural areas of China. The present sample also did not include MSM living with HIV. Second, information and recall bias might exist, e.g., with regards to STI history and the age of first homosexual intercourse. STI history was self-reported in the present study, and the prevalence $(8.2 \%)$ was a bit lower than the data $(11.2 \%)$ reported at the national level [41], probably due to under reporting or low STI screening in Chengdu. Social desirability bias might also exist regarding questions on sexual behaviors. Third, we constructed our own scale, such as positive attitudes towards living with HIV, which should be validated in future studies. Last, we were unable to recruit MSM who were PrEP users, so the association between PrEP usage and disclosure behaviors could not be determined. Most PrEP users in China have so far received PrEP as part of a research trial, but, to our knowledge, no such trials have been conducted in Chengdu.

Despite these limitations, the current study is the first (to our knowledge) to explore disclosure behaviors in the era of $\mathrm{U}=\mathrm{U}$ and PrEP in China. The rates of $\mathrm{U}=\mathrm{U}$ and PrEP awareness were unexpectedly low, 20\% for $\mathrm{U}=\mathrm{U}$ and $51 \%$ for PrEP, indicating huge information gaps among MSM in China.

\section{Conclusion}

Overall, HIV serostatus disclosure was low among this young and relatively highly educated sample of Chinese MSM in the era of $U=U$ and PrEP. The awareness of PrEP and $\mathrm{U}=\mathrm{U}$ was not associated with either informing or asking behaviors. Common barriers to HIV disclosure included perceiving a low risk of HIV infection, engaging in receptive sex, and having sex with casual partners. Having a positive attitude towards living with HIV was associated with always informing partners about one's HIV status. Those who were older than 25 years old or poorly educated, and those earned less, were less likely to disclose their HIV status and might be in particular need for additional support.
Acknowledgements The authors would like to thank all participants who took part in the study and all field workers for their help with data collection. We acknowledge Dr. Ian J Saldanha from Brown University for critically reviewing and editing the manuscript.

Author Contributions WC analyzed the data and drafted the manuscript. AE revised the manuscript substantially. JL and WC designed the study, and PL supervised the data collection. All other authors revised the manuscript and approved the final draft.

Funding The research was supported by the National Natural Science Foundation of China (81803334, 71774178, 71974212), a Major Infectious Disease Prevention and Control of the National Science and Technique Major Project (2018ZX10715004), Science and Technology Planning Project of Guangdong Province (2017A020212006), and Science and Technology Research Project of Guangzhou (201607010332, 201607010368).

Data Availability The data that support the findings of this study are available from the corresponding author upon reasonable request.

Code Availability Not applicable.

\section{Declarations}

Conflict of interest The authors declare no conflict of interest.

Ethics Approval Ethical approval was obtained from the Ethics Committee of Sun Yat-sen University ([2018] 049).

Consent to Participate All participants signed written informed consent before the survey started.

Consent for Publication We confirm that the manuscript has not been submitted to any scientific meeting, foreign language journal, or preprint server. We also confirm that neither the manuscript nor any part of it has been published or is under consideration for publication elsewhere. All authors have read and approved this submitted version of the manuscript.

Open Access This article is licensed under a Creative Commons Attribution 4.0 International License, which permits use, sharing, adaptation, distribution and reproduction in any medium or format, as long as you give appropriate credit to the original author(s) and the source, provide a link to the Creative Commons licence, and indicate if changes were made. The images or other third party material in this article are included in the article's Creative Commons licence, unless indicated otherwise in a credit line to the material. If material is not included in the article's Creative Commons licence and your intended use is not permitted by statutory regulation or exceeds the permitted use, you will need to obtain permission directly from the copyright holder. To view a copy of this licence, visit http://creativecommons.org/licenses/by/4.0/.

\section{References}

1. Serovich JM, Oliver DG, Smith SA, Mason TL. Methods of HIV disclosure by men who have sex with men to casual sexual partners. AIDS Patient Care STDS. 2005;19(12):823-32.

2. Hightow-Weidman LB, Phillips G, Outlaw AY, Wohl AR, Fields $\mathrm{S}$, Hildalgo J, et al. Patterns of HIV disclosure and condom use 
among HIV-infected young racial/ethnic minority men who have sex with men. AIDS Behav. 2013;17(1):360-8.

3. Przybyla SM, Golin CE, Widman L, Grodensky CA, Earp JA, Suchindran C. Serostatus disclosure to sexual partners among people living with HIV: examining the roles of partner characteristics and stigma. AIDS Care. 2013;25(5):566-72.

4. Zhang L, Chow EP, Jing J, Zhuang X, Li X, He M, et al. HIV prevalence in China: integration of surveillance data and a systematic review. Lancet Infect Dis. 2013;13(11):955-63.

5. Bowring A, Veronese V, Doyle J, Stoove M, Hellard M. HIV and sexual risk among men who have sex with men and women in Asia: a systematic review and meta-analysis. AIDS Behav. 2016;20(10):2243-65.

6. Deuba K, Sapkota D, Shrestha U, Shrestha R, Rawal BB, Badal $\mathrm{K}$, et al. Effectiveness of interventions for changing HIV related risk behaviours among key populations in low-income setting: a meta-analysis, 2001-2016. Sci Rep. 2020;10(1):1-13.

7. Tang S, Tang W, Meyers K, Chan P, Chen Z, Tucker JD. HIV epidemiology and responses among men who have sex with men and transgender individuals in China: a scoping review. BMC Infect Dis. 2016;16(1):588.

8. Zhu Z, Yan H, Wu S, Xu Y, Xu W, Liu L, et al. Trends in HIV prevalence and risk behaviours among men who have sex with men from 2013 to 2017 in Nanjing, China: a consecutive crosssectional survey. BMJ Open. 2019;9:1.

9. Xu J-J, Tang W-M, Zou H-C, Mahapatra T, Hu Q-H, Fu G-F, et al. High HIV incidence epidemic among men who have sex with men in china: results from a multi-site cross-sectional study. Infect Dis Poverty. 2016;5(1):82.

10. Sullivan KM. Male self-disclosure of HIV-positive serostatus to sex partners: a review of the literature. J Assoc Nurses AIDS Care. 2005;16(6):33-47.

11. Obermeyer CM, Baijal P, Pegurri E. Facilitating HIV disclosure across diverse settings: a review. Am J Public Health. 2011;101(6):1011-23.

12. Smith R, Rossetto K, Peterson BL. A meta-analysis of disclosure of one's HIV-positive status, stigma and social support. AIDS Care. 2008;20(10):1266-75.

13. Wei C, Lim SH, Guadamuz TE, Koe S. HIV disclosure and sexual transmission behaviors among an Internet sample of HIV-positive men who have sex with men in Asia: implications for prevention with positives. AIDS Behav. 2012;16(7):1970-8.

14. Cohen MS, Chen YQ, McCauley M, Gamble T, Hosseinipour MC, Kumarasamy N, et al. Antiretroviral therapy for the prevention of HIV-1 transmission. N Engl J Med. 2016;375(9):830-9.

15. Rodger AJ, Cambiano V, Bruun T, Vernazza P, Collins S, Van Lunzen J, et al. Sexual activity without condoms and risk of HIV transmission in serodifferent couples when the HIV-positive partner is using suppressive antiretroviral therapy. JAMA. 2016;316(2):171-81.

16. Bavinton BR, Pinto AN, Phanuphak N, Grinsztejn B, Prestage GP, Zablotska-Manos IB, et al. Viral suppression and HIV transmission in serodiscordant male couples: an international, prospective, observational, cohort study. Lancet HIV. 2018;5(8):e438-47.

17. Rodger AJ, Cambiano V, Bruun T, Vernazza P, Collins S, Degen $\mathrm{O}$, et al. Risk of HIV transmission through condomless sex in serodifferent gay couples with the HIV-positive partner taking suppressive antiretroviral therapy (PARTNER): final results of a multicentre, prospective, observational study. The Lancet. 2019;393(10189):2428-38

18. Eisinger RW, Dieffenbach CW, Fauci AS. HIV viral load and transmissibility of HIV infection: undetectable equals untransmittable. JAMA. 2019;321(5):451-2.

19. Okoli C, Van de Velde N, Richman B, Allan B, Castellanos E, Young $B$, et al. Undetectable equals untransmittable $(U=U)$ : awareness and associations with health outcomes among people living with HIV in 25 countries. Sex Transm Infect. 2020. https:// doi.org/10.1136/sextrans-2020-054551.

20. Wang N, Huang B, Ruan Y, Amico KR, Vermund SH, Zheng S, et al. Association between stigma towards HIV and MSM and intimate partner violence among newly HIV-diagnosed Chinese men who have sex with men. BMC Public Health. 2020;20(1):204.

21. Grant RM, Lama JR, Anderson PL, McMahan V, Liu AY, Vargas $\mathrm{L}$, et al. Preexposure chemoprophylaxis for HIV prevention in men who have sex with men. N Engl J Med. 2010;363(27):2587-99.

22. Molina J-M, Capitant C, Spire B, Pialoux G, Cotte L, Charreau I, et al. On-demand preexposure prophylaxis in men at high risk for HIV-1 infection. N Engl J Med. 2015;373:2237-46.

23. Peng L, Cao W, Gu J, Hao C, Li J, Wei D, et al. Willingness to use and adhere to HIV pre-exposure prophylaxis (PrEP) among men who have sex with men (MSM) in China. Int J Environ Res Public Health. 2019;16(14):2620.

24. Chengdu Gay Care Organization. Chengdu Gay Care Organization annual report 2012. 2012.

25. Statistics CBo. Announcement of the Chengdu Bureau of Statistics on the average salary of employees in urban areas of Chengdu in 2019. 2020.

26. Huntingdon B, de Wit J, Duracinsky M, Juraskova I. Belief, covariates, and impact of the "Undetectable $=$ Untransmittable" message among people living with HIV in Australia. AIDS Patient Care STDS. 2020;34(5):205-12.

27. Rendina HJ, Talan AJ, Cienfuegos-Szalay J, Carter JA, Shalhav O. Treatment is more than prevention: perceived personal and social benefits of undetectable $=$ untransmittable messaging among sexual minority men living with HIV. AIDS Patient Care STDS. 2020;34(10):444-51.

28. Carneiro PB, Westmoreland DA, Patel VV, Grov C. Awareness and acceptability of undetectable $=$ untransmittable among a US National Sample of HIV-negative sexual and gender minorities. AIDS Behav. 2020;25:634

29. Lau JT, Wang Z, Kim Y, Li J, Gu J, Mo PK, et al. Low sustainability, poor governance, and other challenges encountered by grassroots non-governmental organizations targeting HIV prevention for men who have sex with men in China-a nation-wide study. AIDS Care. 2017;29(12):1480-90.

30. Zuo L, Liu K, Liu H, Hu Y, Zhang Z, Qin J, et al. Trend of HIV-1 drug resistance in China: a systematic review and meta-analysis of data accumulated over 17 years (2001-2017). E Clin Med. 2020;18:100238.

31. Cao W, Sun S, Peng L, Gu J, Hao C, Li J, et al. Low willingness to pay for pre-exposure prophylaxis (PrEP) among men who have sex with men (MSM) in China. BMC Public Health. 2020;20(1):1-11.

32. Han J, Bouey JZ, Wang L, Mi G, Chen Z, He Y, et al. PrEP uptake preferences among men who have sex with men in China: results from a National Internet Survey. J Int AIDS Soc. 2019;22(2):25242.

33. Eaton LA, Kalichman SC, Cherry C. Sexual partner selection and HIV risk reduction among Black and White men who have sex with men. Am J Public Health. 2010;100(3):503-9.

34. Murphy DA, de Wit JB, Donohoe S, Adam PC. The need to know: HIV status disclosure expectations and practices among non-HIV-positive gay and bisexual men in Australia. AIDS Care. 2015;27(sup1):90-8.

35. Marcus U, Gassowski M, Drewes J. HIV risk perception and testing behaviours among men having sex with men (MSM) reporting potential transmission risks in the previous 12 months from a large online sample of MSM living in Germany. BMC Public Health. 2016;16(1):1111.

36. Chu J-H, Huang J-H. Psychosociobehavioral characteristics associated with high condomless anal intercourse intention: a 
comparison of receptive, versatile, and insertive MSM in Taiwan. AIDS Care. 2019;30:770.

37. Meng X, Zou H, Fan S, Zheng B, Zhang L, Dai X, et al. Relative risk for HIV infection among men who have sex with men engaging in different roles in anal sex: a systematic review and meta-analysis on global data. AIDS Behav. 2015;19(5):882-9.

38. Yang H, Ye L, Su L, Liu Y, Xiao L, Hu Y, et al. An analysis on incidence of HIV-1 epidemics among men who have sex with men in Sichuan Province during 2011-2015. Zhonghua Yu Fang Yi Xue Za Zhi. 2019;53(3):327-9.

39. Duan Z, Shuangfeng F, Rong L, Xueqing W, Yaying S, Zhijun L, et al. Consistently high HIV prevalence among men who have sex with men in Chengdu city from 2009 to 2014. Int J STD AIDS. 2016;27(12):1057-62.
40. Yan H, Cao W, Mo P, Huan X, Wang Z, Lin X, et al. Prevalence and associated factors of HIV serostatus disclosure to regular female sex partners among HIV-positive men who have sex with both men and women in China. AIDS Care. 2019;31(8):1026-34.

41. Zhou Y, Li D, Lu D, Ruan Y, Qi X, Gao G. Prevalence of HIV and syphilis infection among men who have sex with men in China: a meta-analysis. BioMed Res Int. 2014;2014:620431.

Publisher's Note Springer Nature remains neutral with regard to jurisdictional claims in published maps and institutional affiliations. 